\title{
The Association between Vaccination and Autoimmunity
}

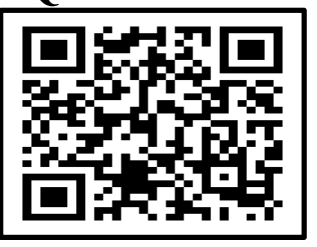

MEENAKSHI CHOPRA', YAGYESHWAR MALHOTRA*2, VARSHA SHARMA³, SOURAV CHANDRA BIDYASAGAR BAL ${ }^{4}$

Vaccines are one of the prime preventive measures against infectious diseases. Administration of vaccination may lead to significant autoimmune manifestations. There is a vast literature on autoimmune issues associated with vaccines including animal and in-vitro studies, case reports and case series. Contrary to this, epidemiological research work does not report this association. This review on the potential association between vaccines and the development of autoimmune diseases is based on currently available scientific literature. We conclude that vaccines have a very positive impact on human health. Further research is required to clarify the association between vaccines and autoimmune conditions and also the detailed mechanisms behind this.

KEYWORDS: Vaccines, Vaccination, Autoimmunity

\section{INTRODUCTION}

The demonstrated association between infection and autoimmunity in genetically predisposed individuals ${ }^{1}$, has opened the debate in the scientific community about the possibility that vaccines may also induce autoimmune phenomena. ${ }^{2}$ There are numerous reports of patients who have developed autoimmune diseases after certain vaccinations, which raises the suspicion of a causal relationship, but there are several epidemiological studies that have not confirmed this association, which has led to intense debate on this topic. ${ }^{3}$ The possibility that a vaccine is capable of inducing an autoimmune event must not be overlooked, although the magnitude of the potential risk is apparently low. There is currently still limited knowledge related to the etiology, genetic basis and other risk factors that are part of the mosaic of autoimmune diseases, so this is a field of intense research followed closely by health and regulatory entities in all countries.

There are various models of autoimmune diseases, induced in laboratory animals by immunization with antigens obtained from their own tissues formulated with adjuvants. A classic example is the experimental allergic encephalitis model, which is obtained in susceptible rat and mouse lines, by inoculating tissue extracts from the central nervous system, myelin basic protein, or peptides derived from it with Freund's Complete Adjuvant, resulting in characteristic neurological symptoms. ${ }^{4}$ Other models have been described to reproduce an autoimmune disease in laboratory animals, following similar protocols. ${ }^{5}$ However, it has been described that inoculation of a peptide from the hepatitis B virus polymerase, which shares sequential similarities to rabbit MBP, by being inoculated with this, produced perivascular infiltration localized in the central nervous system, similar to that occurring with inoculation of full-length MBP or encephalitogenic peptides derived from it, further demonstrating a humoral and cellular immune response against MBP itself. $^{6}$

Experimental models provide a basis for studying the mechanisms of a potential development of postvaccine autoimmune disease. However, it should be considered that such models do not reproduce exactly what happens when a vaccine is given in humans, since they are based on immunization regimens under extreme conditions, using adjuvants such as Freund's, not approved for clinical use; in addition, genetically susceptible models are almost always used and immunized with purified organ or self-antigen homogenates, which does not occur in practice. There are numerous suspected cases of post-vaccination autoimmune diseases documented in the medical literature. Similarly, there are periodic databases and publications that record adverse events associated with vaccines that allow the causal relationship between vaccines and their adverse effects to be processed and statistically analyzed, including postvaccination autoimmune events. ${ }^{7}$ The first case of 
suspected association of hepatitis B vaccines with signs of central nervous system demyelination in humans was recorded in $1991 .{ }^{8}$ Then, in France, 35 cases of demyelinating diseases were described, occurring within eight weeks after a similar vaccination was given, with half of the cases being confirmed as multiple sclerosis. ${ }^{9}$ The accumulation of spontaneous multiple sclerosis cases led the French Minister of Public Health to temporarily prohibit the vaccination program of schoolchildren with the hepatitis B vaccine initiated in 1995, which coupled with the absence of epidemiological data demonstrating otherwise, created much debate and confusion. ${ }^{10}$ Epidemiological studies and other analyses were subsequently performed that could not confirm this causal relationship. ${ }^{11,12}$

Girard has questioned the methods used and the conclusions issued after the controlled studies, highlighting that not all cases of autoimmunity associated with this vaccine were collected, and the inappropriateness of the statistical methods used. ${ }^{13}$ However, Bogdanos et al. reported that several patients vaccinated against hepatitis B developed antibodies against myelin oligodendrocyte protein mimetic peptides; however, no clinical consequences of this cross-reactivity were detected and the antibodies decreased their titer in the subsequent months. No anti-MBP peptide antibodies ${ }^{14}$ were detected in this same study. This supports the criterion that an autoimmune reaction does not always have a clinical translation, which further complicates interpretation of the results. The actual danger of hepatitis B vaccination is still debated, and it is generally accepted that if a true causal relationship exists in practice, the benefits reported for this vaccine outweigh the potential risk. The existence of autoimmune phenomena associated with natural hepatitis A infection is known, but in relation to hepatitis A vaccines, to date, one case of a possible connection to autoimmune hepatitis has been reported, ${ }^{15}$ so this vaccine is considered one of the safest, after millions of doses given worldwide.

Guillain-Barré syndrome also called demyelinating polyradiculoneuritis, is closely related to a previous infection, with evidence of cross-immune reaction between viral antigens and myelinic antigens and constituting the most common autoimmune condition that has been related to influenza vaccines. ${ }^{16}$ The relationship of GBS to influenza A/New Jersey vaccine was suggested following a vaccination campaign conducted in the United States between 1976 and 1977, during which the number of cases of this condition increased in the first weeks after vaccination, particularly in the fifth week, with the incidence decreasing after the sixth week, leading to a temporary suspension of the vaccination program. ${ }^{17}$

However, retrospective research developed after campaigns conducted in 1992-1993 and 1993-1994 revealed that the incidence within six weeks was significant although much lower than in the campaign of the $1970{ }^{.18}{ }^{18}$ Another found a significantly increased risk of developing GBS following influenza vaccination, with differences between vaccine producers and the viral strains used. ${ }^{19}$ Meanwhile, it has been shown that the risk of GBS recurrence following an initial episode and re-immunization with influenza vaccine is minimal. Influenza vaccine may also result in Henoch-Schönlein purpura, microscopic polyangiitis, reactive arthritis, giant cell vasculitis, polymyalgia rheumatica, cryoglobulinemia, polyarthralgia, fatigue, meningoencephalitis/encephalitis, optic neuritis, transverse myelitis, brachial neuritis, Guillain-Barré syndrome, Bell's palsy, lymphocytopenia, thrombocytopenia, autoimmune hemolytic anemia, acute myocarditis, acute pericarditis, type 1 diabetes mellitus, nephrotic syndrome, uveitis, myositis, erythema multiforme.

It has been suggested that immunization with $\mathrm{H}$. influenzae type $b$ vaccine after the age of two months may be related to type 1 diabetes in humans and that the potential risk of the vaccine may exceed the benefit it provides. ${ }^{20}$ However, this association has not been confirmed in controlled studies.(21) Hepatitis B vaccine might result in rheumatoid arthritis, reactive arthritis, SLE exacerbation, Sjogren's syndrome, vasculitis, cryoglobulinemia, polyarthralgia, polyarteritis nodosa, myalgia, fatigue, erythema nodosum, erythema multiforme, cutaneous lupus erythematosus, localized scleroderma, lichen planus, encephalitis, acute aseptic meningitis, transverse myelitis, optic neuritis, neuropathy of lumbar and brachial plexuses, Guillain-Barré syndrome, Bell's palsy, acute cerebellar ataxia, myasthenia gravis, multiple sclerosis, thrombocytopenia, immune hemolytic anemia, aplastic anemia, nephrotic syndrome, uveitis, alopecia, acute pericarditis and Graves' disease. The re-emergence of yellow fever has led to mass vaccination in countries such as Brazil, Australia, Spain, among others, with attenuated vaccines such 
as ${ }_{17} \mathrm{D}$ and ${ }_{17} \mathrm{DD}$, which have been very successful in their protective capacity; however, the occurrence of very sporadic conditions called viscerotropic and neurotropic diseases, have led to discussions about its safety. ${ }^{22}$ The mechanism of these severe adverse effects produced by these vaccines ${ }^{23}$ has not yet been determined, although it appears that people over 60 years of age, and those with some predisposition or immunological compromise, exhibit an increased risk of these adverse events, so it is proposed to evaluate the risk/benefit of vaccination ${ }^{24}$ in these populations. These vaccines are generally considered safe and highly effective against this dangerous disease. Measles Mumps Rubella vaccine can cause arthritis and acute arthralgia, chronic arthritis, myositis, encephalitis, aseptic meningitis, myelitis, optic neuritis, Guillain-Barré syndrome, thrombocytopenia/acute thrombocytopenic purpura, hemolytic uremic syndrome, hemolytic anemia. Varicella vaccine is known to be associated with arthritis or arthralgia, vasculitis, encephalitis, aseptic meningitis, myelitis, optic neuritis, Guillain-Barré syndrome, transient cerebellar ataxia, optic neuritis, transverse myelitis, Bell's palsy, thrombocytopenia, aplastic anemia, erythema multiforme. Poliomyelitis vaccine may induce acute arthritis, encephalitis, Guillain-Barré syndrome and autoimmune hemolytic anemia. Smallpox vaccine may cause post-vaccine encephalopathy, encephalomyelitis, erythema multiforme, myopericarditis, dilated cardiomyopathy. Diphtheria/tetanus toxoid is known to be associated with demyelinating diseases of the central nervous system and Guillain-Barré syndrome. BCG vaccine can cause Arthritis and Reiter's disease.

A latent concern of the health and regulatory authorities is the possibility that a vaccine may aggravate a pre-existing autoimmune disease. In this regard, several controlled studies, particularly in patients with multiple sclerosis, rheumatoid arthritis and SLE, using hepatitis B, influenza, pneumococcal and tetanus toxoid vaccines have not revealed evidence of worsening of the disease postvaccination, ${ }^{10}$ so there are no objective criteria to date that a patient-carrier of autoimmune disease must not be vaccinated. The development of an autoimmune process rests on the influence of genetic factors associated with external factors that induce or facilitate the loss of immunotolerance with the development of immune responses against its own structures. The genetic factor determines that not all individuals have the same predisposition to develop an autoimmune disease, among the most commonly involved genes are those that code for Human Leucocyte Antigens. There are reports of association of certain HLA haplotypes with autoimmune diseases, with HLA-B27 being the haplotype most associated with diseases such as ankylosing spondylitis. ${ }^{25}$ Other genes recently associated with autoimmune diseases are the autoimmune regulator genes, which participates in the presentation of selfantigens during the central tolerance process in the thymus, for the deletion of autoreactive T-cells, the defect of which may lead to the development of multisystemic autoimmune diseases. ${ }^{26}$ Associations with polymorphisms have also been found in the gene encoding the cytolytic $\mathrm{T}$ lymphocyte associated antigen, a negative regulator in T-cell activation, and in the TIM (T-cell immunoglobulin and mucindomain-containing) family of proteins. ${ }^{27}$ Molecular mimicry in microbial antigens may constitute a potential hazard for the development of post-vaccine autoimmunity, ${ }^{1,6}$ especially in the presence of a vaccine adjuvant. Adjuvants optimize the immune response against the co-administered antigen, but also contribute to vaccine toxicity, ${ }^{28}$ so there is a hypothesis that certain autoreactive lymphocyte clones, which, under physiological conditions, do not recognize self-antigens due to being under the peripheral tolerance pressure, could well increase their ability to recognize and activate through the influence of adjuvant activity and develop an autoreactive response, especially in individuals with a favorable genetic background. ${ }^{29}$ Fortunately, the tolerance mechanisms are very effective in limiting the deleterious effect that molecular mimicry could cause between microorganisms and their own structures. Another mechanism that may act on postvaccine autoimmunity is the formation of immune complexes, causing vasculitis or exacerbation of latent autoimmune processes. ${ }^{10}$

Lastly, it should be noted that within the dynamics of the immune response, usually responses against their own structures develop in a subclinical manner whose etiopathogenetic role is not yet known. Although there is theoretically the possibility of induction of post-vaccination autoimmune disease and the existence of documented suspicions of associations between certain vaccines and autoimmune conditions, epidemiological studies have mostly concluded that there is no such association or is of very low incidence. However, the epidemiological research required in vaccines currently has some issues, such as the lack of knowledge of the genetic profile of each vaccinated individual, since all genes 
involved in autoimmunity have not yet been accurately defined. Another difficulty in the interpretation of population-based studies is that the timing of developing an autoimmune response is unpredictable. There is also a need to establish ways to improve the recording of adverse events in the pharmacovigilance studies, since these are performed through passive surveillance in which the patient spontaneously describes the event, which implies the problem that many people do not report and, furthermore, it is more difficult to establish a possible causal relationship with an event that occurs later as time passes after vaccination. All of this implies that refining analysis methods and having new elements in the pathogenesis of autoimmune diseases are necessary in order to establish the correct estimates.

\section{CONCLUSION}

Due to the great benefit to humanity achieved with vaccines, there is no justification for stopping or decreasing their administration. However, the potential risk of post-vaccine autoimmunity cannot be ignored and, although a clear causal relationship has not been established in practice, due to the ethical and medical-legal implications, it is a responsibility of medical field to continue refining evaluation methods from base level to pharmacovigilance studies and try to avoid potential adverse effects as much as possible, with greater rigor in the design and evaluation of vaccine candidates under development.

\section{REFERENCES}

1. Rose NR. Autoimmunity, infections and adjuvants. Lupus. 2010;19(4):354-8.

2. Orbach H, Shoenfeld Y. Vaccination, infection and autoimmunity: myth and reality VIAMR 2005-10-2628, Beau-Rivage Palace Hotel, Lausanne, Switzerland. Autoimmunity Rev. 2007(6):261-6.

3. Chen RT, Pless R, DeStefano F. Epidemiology of autoimmune reactions induced by vaccination. J Autoimmun. 2001;16(3):309-18.

4. Alvord EC, Kies MW, Suckling AJ. Experimental allergic encephalomyelitis: a useful model for multiple sclerosis. In : Alvord EC, Kies MW, Suckling AJ (Eds). Progress in Clinical and Biological Research. New York; Alan R Liss Inc; 1984. p. 523-37.

5. Burkhardt H, Kalden JR. Animal models of autoimmune diseases. Rheumatol Int. 1997;17(3):91-9. 6. Oldstone MB. Molecular mimicry and immunemediated diseases. FASEB J. 1998;12(13):1255-65.

7. Geier DA, Geier MR. Hepatitis B vaccination and arthritic adverse reactions: a follow-up analysis of the
Vaccine Adverse Events Reporting System (VAERS) database. Clin Exp Rheumatol. 2002;20(1):119.

8. Herroelen L, de Keyser J, Ebinger G. Centralnervoussystem demyelination after immunisation with recombinant hepatitis B vaccine. Lancet. 1991;338(8776):1174-5.

9. Gout O, Lyon-Caen O. Sclerotic plaques and vaccination against hepatitis B. Rev Neurol (Paris). 1998;154(3):205-7.

10. Vial T, Descotes J. Autoimmune diseases and vaccinations. Eur J Dermatol. 2004;14(2):86-90.

11. Duclos P. Safety of immunization and adverse events following vaccination against hepatitis $B$. Expert Opin Drug Saf. 2003;2(3):225-31.

12. Schattner A. Consequence or coincidence? The occurrence, pathogenesis and significance of autoimmune manifestations after viral vaccines. Vaccine. 2005;23(30):3876-86.

13. Girard M. Autoimmune hazards of hepatitis B vaccine. Autoimmun Rev. 2005;4(2):96-100.

14. Bogdanos DP, Smith H, Ma Y, Baum H, MieliVergani G, Vergani D. A study of molecular mimicry and immunological cross-reactivity between hepatitis B surface antigen and myelin mimics. Clin Dev Immunol. 2005;12(3):217-24.

15. Vento S, Garofano T, Di Perri G, Dolci L, Concia E, Bassetti D. Identification of hepatitis A virus as a trigger for autoimmune chronic hepatitis type 1 in susceptible individuals. Lancet. 1991;337(8751):1183-7. 16. Haber P, DeStefano F, Angulo FJ, Iskander J, Shadomy SV, Weintraub E, et al. Guillain-Barré syndrome following influenza vaccination. JAMA. 2004;292(20):2478-81.

17. Schoenberger LB, Bregman DJ, Sullivan-Bolyai JZ, Keenlyside RA, Ziegler DW, Retailliau HF, et al. Guillain-Barre syndrome following vaccination in the National Influenza Immunization Program, United States, 1976-1977. Am J Epidemiol. 1979;110(2):105-23. 18. Lasky T, Terracciano Gj, Madger L, Koski CL, Ballesteros M, Nash D, et al. The Guillain-Barré syndrome and the 1992-1993 and 1993-1994 influenza vaccines. N Engl J Med. 1998;339(25):1797-802.

19. Geier MR, Geier DA, Zahalsky AC. Influenza vaccination and Guillain Barre syndrome small star, filled. Clin Immunol. 2003;107(2):116-21.

20. Childhood immunizations and type 1 diabetes: summary of an Institute for Vaccine Safety Workshop. The Institute for Vaccine Safety Diabetes Workshop Panel. Pediatr Infect Dis J. 1999;18(3):21722.

21. Karvonen M, Cepaitis Z, Tuomilehto J. Association between type 1 diabetes and Haemophilus influenzae 
type b vaccination: birth cohort study. BMJ. 1999;318(7192):1169-72.

22. Thomas RE, Lorenzetti DL, Spragins W, Jackson $\mathrm{D}$, Williamson T. Active and passive surveillance of yellow fever vaccine $17 \mathrm{D}$ or $17 \mathrm{DD}$-associated serious adverse events: systematic review. Vaccine. 2011;29(28):4544-55.

23. Barrett AD, Teuwen DE. Yellow fever vaccine how does it work and why do rare cases of serious adverse events take place? Curr Opin Immunol. 2009;21(3):308-13.

24. Khromava AY, Eidex RB, Weld LH, Kohl KS, Bradshaw RD, Chen RT, et al. Yellow fever vaccine: an updated assessment of advanced age as a risk factor for serious adverse events. Vaccine. 2005;23(25):325663.

25. Braun J, Bollow M, Remlinger G, Eggens U, Rudwaleit M, Distler A, et al. Prevalence of spondyloarthropathies in HLA-B27 positive and negative blood donors. Arthritis Rheum. 1998; 41(1):58-67.

26. Pitkanen J, Peterson P. Autoimmune regulator: from loss of function to autoimmunity. Genes Immun. 2003;4(1):12-21.

27. Anderson AC, Anderson DE. TIM-3 in autoimmunity. Curr Opin Immunol. 20o6;18(6):6659.

28. Batista-Duharte A, Lindblad EB, Oviedo-Orta E. Progress in understanding adjuvant immunotoxicity mechanisms. Toxicol Lett. 2011;203(2):97-105.

29. Waisbren BA Sr. Acquired autoimmunity after viral vaccination is caused by molecular mimicry and antigen complimentarity in the presence of an immunologic adjuvant and specific HLA patterns. Med Hypotheses. 2008;70(2):346-8.
Cite this article as:

Chopra M, Malhotra Y, Sharma V, Bal SCB. The Association between Vaccination and Autoimmunity. Int Healthc Res J. 2021;5(2):RV9-RV13. https://doi.org/10.26440/IHRJ/0502.05422

AUTHOR AFFILIATIONS: (*Corresponding Author)

1. Reader, Department of Public Health Dentistry, SGT Dental College, Hospital and Research Institute, SGT University, Gurugram

2. MDS (Orthodontics and Dentofacial Orthopedics), Consultant Orthodontist, Kangra, HP, India (Corresponding Author)

3. Reader, Public Health Dentistry, Mansarovar Dental College, Kolar Road, Bhopal

4. Department of Public Health Dentistry, Institute of Dental Science, SOA University Bhubaneswar 